\title{
An Erdős-Ko-Rado theorem for multisets
}

\author{
Karen Meagher * \\ Department of Mathematics and Statistics \\ University of Regina, Regina, Saskatchewan, Canada \\ kmeagher@math.uregina.ca \\ Alison Purdy \\ Department of Mathematics and Statistics \\ University of Regina, Regina, Saskatchewan, Canada \\ purdyali@math.uregina.ca
}

Submitted: Aug 9, 2011; Accepted: Oct 26, 2011; Published: Nov 21, 2011

Mathematics Subject Classification: 05D05

\begin{abstract}
Let $k$ and $m$ be positive integers. A collection of $k$-multisets from $\{1, \ldots, m\}$ is intersecting if every pair of multisets from the collection is intersecting. We prove that for $m \geq k+1$, the size of the largest such collection is $\left(\begin{array}{c}m+k-2 \\ k-1\end{array}\right)$ and that when $m>k+1$, only a collection of all the $k$-multisets containing a fixed element will attain this bound. The size and structure of the largest intersecting collection of $k$-multisets for $m \leq k$ is also given.
\end{abstract}

\section{Introduction}

The Erdös-Ko-Rado Theorem [6] is an important result in extremal set theory that gives the size and structure of the largest pairwise intersecting $k$-subset system from $[n]=$ $\{1, \ldots, n\}$. This theorem is commonly stated as follows:

Theorem 1.1. Let $k$ and $n$ be positive integers with $n \geq 2 k$. If $\mathcal{F}$ is a collection of intersecting $k$-subsets of $[n]$, then

$$
|\mathcal{F}| \leq\left(\begin{array}{l}
n-1 \\
k-1
\end{array}\right)
$$

Moreover, if $n>2 k$, equality holds if and only if $\mathcal{F}$ is a collection of all the $k$-subsets from $[n]$ that contain a fixed element from $[n]$.

\footnotetext{
${ }^{*}$ Research supported by NSERC.
} 
Note that if $n<2 k$, any pair of $k$-subsets will be intersecting and so the largest intersecting collection will have size $\left(\begin{array}{l}n \\ k\end{array}\right)$.

A multiset is a generalization of a set in which an element may appear more than once. As with sets, the order of the elements is irrelevant. The cardinality of a multiset is the total number of elements including repetitions. A $k$-multiset system on an $m$-set is a collection of multisets of cardinality $k$ containing elements from $[m]$. We say that two multisets are intersecting if they have at least one element in common and that a collection of multisets is intersecting if every pair of multisets in the collection is intersecting.

In this paper, we give a generalization of the Erdős-Ko-Rado Theorem to intersecting multiset systems. Specifically, we prove the following two theorems for the cases when $m \geq k+1$ and $m \leq k$ respectively.

Theorem 1.2. Let $k$ and $m$ be positive integers with $m \geq k+1$. If $\mathcal{A}$ is a collection of intersecting $k$-multisets of $[m]$, then

$$
|\mathcal{A}| \leq\left(\begin{array}{c}
m+k-2 \\
k-1
\end{array}\right)
$$

Moreover, if $m>k+1$, equality holds if and only if $\mathcal{A}$ is a collection of all the $k$-multisets from $[m]$ that contain a fixed element from $[\mathrm{m}]$.

If $m<k+1$, larger collections are possible. For example, if $m=k=3$, the seven $k$ multisets containing either two or three distinct elements from $[m]$ will form an intersecting collection since each multiset contains more than half the elements from $[m]$. We will use $\mathcal{M}_{\left(>\frac{m}{2}\right)}$ to denote the collection of all $k$-multisets that contain more than $\frac{m}{2}$ distinct elements from $[m]$ and $\mathcal{M}_{\left(\frac{m}{2}\right)}$ to denote the collection of all $k$-multisets from $[m]$ containing exactly $\frac{m}{2}$ distinct elements. Then

$$
\left|\mathcal{M}_{\left(\frac{m}{2}\right)}\right|=\left(\begin{array}{c}
m \\
\frac{m}{2}
\end{array}\right)\left(\begin{array}{c}
k-1 \\
k-\frac{m}{2}
\end{array}\right)
$$

and

$$
\left|\mathcal{M}_{\left(>\frac{m}{2}\right)}\right|=\sum_{j=\left\lceil\frac{m+1}{2}\right\rceil}^{m}\left(\begin{array}{c}
m \\
j
\end{array}\right)\left(\begin{array}{l}
k-1 \\
k-j
\end{array}\right) .
$$

Theorem 1.3. Let $k$ and $m$ be positive integers with $m \leq k$. If $\mathcal{A}$ is a collection of intersecting $k$-multisets of $[m]$, then:

1. If $m$ is odd, $|\mathcal{A}| \leq\left|\mathcal{M}_{\left(>\frac{m}{2}\right)}\right|$ and equality holds if and only if $\mathcal{A}=\mathcal{M}_{\left(>\frac{m}{2}\right)}$.

2. If $m$ is even, $|\mathcal{A}| \leq\left|\mathcal{M}_{\left(>\frac{m}{2}\right)}\right|+\frac{1}{2}\left|\mathcal{M}_{\left(\frac{m}{2}\right)}\right|$ and equality holds if and only if $\mathcal{A}$ consists of $\mathcal{M}_{\left(>\frac{m}{2}\right)}$ and a maximal intersecting collection of $k$-multisets from $\mathcal{M}_{\left(\frac{m}{2}\right)}$. 
A $k$-multiset on an $m$-set can be represented as an integer sequence of length $m$ with the integer in each position representing the number of repetitions of the corresponding element from $[m]$. For example, if $m=6$, the multiset $\{1,2,2,4\}$ can be represented by the integer sequence $(1,2,0,1,0,0)$. For a $k$-multiset, the sum of the integers in the corresponding integer sequence will equal $k$.

Erdős-Ko-Rado type results for intersecting families of integer sequences are known (e.g. [9], [10], [11]). In these, the sum of the entries in the integer sequence is not restricted to $k$ and the definition of intersection is different from our definition for multisets. In [2], Anderson proves an Erdős-Ko-Rado type result for multisets but uses yet another definition of intersection. A definition of intersection equivalent to ours is used in several theorems for intersecting collections of vectors presented by Anderson in [3]. These theorems were originally written in terms of sets of noncoprime divisors of a number by Erdös et al. in [5] and [7], and again the sum of the entries is not restricted to $k$.

More recently, Brockman and Kay [4] proved the result in Theorem 1.2 provided that $m \geq 2 k$. Mahdian [13] proved the bound on the size of a collection of intersecting $k$ multisets when $m>k$ using a method similar to Katona's cycle proof for sets [12]. Our results improve the bound on $m$ given in [4] and give the size and structure of the largest possible intersecting collection for all values of $m$ and $k$.

\section{Proof of Theorem 1.2}

Our proof of this theorem uses a homomorphism from a Kneser graph to a graph whose vertices are the $k$-multisets of $[m]$.

A Kneser graph, $K(n, k)$, is a graph whose vertices are all of the $k$-sets from an $n$-set, denoted by $\left(\begin{array}{c}{[n]} \\ k\end{array}\right)$, and where two vertices are adjacent if and only if the corresponding $k$ sets are disjoint. Thus an independent set of vertices in the Kneser graph is an intersecting $k$-set system. We will use $\alpha(K(n, k))$ to denote the size of the largest independent set in $K(n, k)$.

We now define a multiset analogue of the Kneser graph. For positive integers $k$ and $m$, let $M(m, k)$ be the graph whose vertices are the $k$-multisets from the set $[m]$, denoted by $\left(\left(\begin{array}{c}{[m]} \\ k\end{array}\right)\right)$, and where two vertices are adjacent if and only if the corresponding multisets are disjoint. For this graph, the number of vertices is equal to $\left(\left(\begin{array}{c}m \\ k\end{array}\right)\right)=\left(\begin{array}{c}m+k-1 \\ k\end{array}\right)$ and an independent set is an intersecting $k$-multiset system.

Let $n=m+k-1$. Then $K(n, k)$ has the same number of vertices as $M(m, k)$ and $B \cap[m] \neq \emptyset$ for any $B \in\left(\begin{array}{c}{[n]} \\ k\end{array}\right)$.

For a set $A \subseteq[m]$ of cardinality $a$ where $1 \leq a \leq k$, the number of $k$-sets, $B$, from $[n]$ such that $B \cap[m]=A$ will be equal to

$$
\left(\begin{array}{c}
n-m \\
k-a
\end{array}\right)=\left(\begin{array}{c}
k-1 \\
k-a
\end{array}\right) .
$$

Similarly, the number of $k$-multisets from $[m]$ that contain all of the elements of $A$ and 
no others will be equal to

$$
\left(\left(\begin{array}{c}
a \\
k-a
\end{array}\right)\right)=\left(\begin{array}{c}
a+(k-a)-1 \\
k-a
\end{array}\right)=\left(\begin{array}{l}
k-1 \\
k-a
\end{array}\right) .
$$

Hence there exists a bijection, $f:\left(\begin{array}{c}{[n]} \\ k\end{array}\right) \rightarrow\left(\left(\begin{array}{c}{[m]} \\ k\end{array}\right)\right)$, such that for any $B \in\left(\begin{array}{c}{[n]} \\ k\end{array}\right)$, the set of distinct elements in $f(B)$ will be equal to $B \cap[m]$.

If $A, B \in\left(\begin{array}{c}{[n]} \\ k\end{array}\right)$ are two adjacent vertices in the Kneser graph, then $(A \cap[m]) \cap(B \cap[m])=$ $\emptyset$ and hence $f(A) \cap f(B)=\emptyset$. Therefore $f(A)$ is adjacent to $f(B)$ if $A$ is adjacent to $B$ and so the bijection $f:\left(\begin{array}{c}{[n]} \\ k\end{array}\right) \rightarrow\left(\left(\begin{array}{c}{[m]} \\ k\end{array}\right)\right)$ is a graph homomorphism. In fact, $K(n, k)$ is isomorphic to a spanning subgraph of $M(m, k)$. Thus

$$
\alpha(M(m, k)) \leq \alpha(K(n, k)) .
$$

From the Erdős-Ko-Rado Theorem, we have that if $n \geq 2 k$,

$$
\alpha(K(n, k))=\left(\begin{array}{l}
n-1 \\
k-1
\end{array}\right) .
$$

Thus, for $m \geq k+1$,

$$
\alpha(M(m, k)) \leq\left(\begin{array}{l}
n-1 \\
k-1
\end{array}\right)=\left(\begin{array}{c}
m+k-2 \\
k-1
\end{array}\right)
$$

An intersecting collection of $k$-multisets from $[m]$ consisting of all $k$-multisets containing a fixed element from $[m]$ will have size $\left(\begin{array}{c}m+(k-1)-1 \\ k-1\end{array}\right)=\left(\begin{array}{c}m-k-2 \\ k-1\end{array}\right)$. Therefore

$$
\alpha(M(m, k))=\left(\begin{array}{c}
m+k-2 \\
k-1
\end{array}\right)
$$

which gives the upper bound on $\mathcal{A}$ in Theorem 1.2.

To prove the uniqueness statement in the theorem, let $m>k+1$ and let $\mathcal{A}$ be an intersecting multiset system of size $\left(\begin{array}{c}m+k-2 \\ k-1\end{array}\right)$. With the homomorphism defined above, the pre-image of $\mathcal{A}$ will be an independent set in $K(n, k)$ of size $\left(\begin{array}{l}n-1 \\ k-1\end{array}\right)$. Since $m>k+1$ and $n=m+k-1$, it follows that $n>2 k$ so, by the Erdös-Ko-Rado theorem, $f^{-1}(\mathcal{A})$ will be a collection of all the $k$-subsets of $[n]$ that contain a fixed element from $[n]$. If the fixed element, $x$, is an element of $[m]$, then it follows from the definition of $f$ that every multiset in $\mathcal{A}$ will contain $x$. Thus $\mathcal{A}$ will be a collection of all the $k$-multisets from $[m]$ that contain a fixed element from $[m]$ as required. If $x \notin[m]$, then $f^{-1}(\mathcal{A})$ will include the sets $A=\{1, m+1, \ldots, n\}$ and $B=\{2, m+1, \ldots, n\}$ since $m>k+1$ implies that $m>2$. But $f(A) \cap f(B)=\emptyset$ which contradicts our assumption that $\mathcal{A}$ is an intersecting collection of multisets. Therefore, when $m>k+1$, if $\mathcal{A}$ is an intersecting collection of multisets of the maximum possible size, then $\mathcal{A}$ is the collections of all $k$-multisets containing a fixed element from $[m]$.

The case when $m=k+1$ is analogous to the case when $n=2 k$ in the Erdős-Ko-Rado theorem. The size of the largest possible intersecting collection is equal to $\left(\begin{array}{c}m+k-2 \\ k-1\end{array}\right)$ but collections attaining this bound are not limited to those having a common element in all $k$-multisets. 


\section{Proof of Theorem 1.3}

Although Theorem 1.2 is restricted to $m \geq k+1$, the inequality $\alpha(M(m, k)) \leq \alpha(K(n, k))$ still holds when $m \leq k$. However, the resulting inequality

$$
\alpha(M(m, k)) \leq\left(\begin{array}{l}
n \\
k
\end{array}\right)=\left(\begin{array}{c}
m+k-1 \\
k
\end{array}\right)
$$

is not particularly useful since for $m>1$ this bound is not attainable. Clearly, two multisets consisting of $k$ copies of different elements from $[m]$ will not intersect.

Before proceeding with our proof of Theorem 1.3, we define the support of a multiset. If $A$ is a $k$-multiset from $[m]$, the support of $A$, denoted by $S_{A}$, is the set of distinct integers from $[m]$ in $A$. Note that two $k$-multisets, $A, B \in\left(\left(\begin{array}{c}{[m]} \\ k\end{array}\right)\right)$, will be intersecting if and only if $S_{A} \cap S_{B} \neq \emptyset$ and that each $S_{A}$ will have a unique complement, $\overline{S_{A}}=[m] \backslash S_{A}$, in $[m]$.

Let $\mathcal{A}$ be an intersecting family of $k$-multisets of $[m]$ of maximum size and let $M \in \mathcal{A}$ be a $k$-multiset such that $\left|S_{M}\right|=\min \left\{\left|S_{A}\right|: A \in \mathcal{A}\right\}$. If $m=2$, it is easy to see that the theorem holds, so we will assume that $m>2$.

Suppose that $\left|S_{M}\right|<\frac{m}{2}$. Let $\mathcal{B}_{1}=\left\{A \in \mathcal{A}: S_{A}=S_{M}\right\}$ and let $\mathcal{B}_{2}=\left\{B \in\left(\left(\begin{array}{c}{[m]} \\ k\end{array}\right)\right)\right.$ : $\left.S_{B}=\overline{S_{M}}\right\}$. Then $\mathcal{B}_{1} \subseteq \mathcal{A}$ and $\mathcal{B}_{2} \cap \mathcal{A}=\emptyset$.

We will now show that $\mathcal{A}^{\prime}:=\left(\mathcal{A} \backslash \mathcal{B}_{1}\right) \cup \mathcal{B}_{2}$ is an intersecting family of $k$-multisets from $[m]$ that is larger than $\mathcal{A}$. By construction, every multiset in $\mathcal{A} \backslash \mathcal{B}_{1}$ contains at least one element from $[m] \backslash S_{M}$, and $[m] \backslash S_{M}=S_{B}$ for all $B \in \mathcal{B}_{2}$. Thus $\mathcal{A}^{\prime}$ is an intersecting collection of $k$-multisets.

Let $\left|S_{M}\right|=i$. Then

$$
\left|\mathcal{B}_{1}\right|=\left(\left(\begin{array}{c}
i \\
k-i
\end{array}\right)\right)=\left(\begin{array}{c}
k-1 \\
k-i
\end{array}\right) .
$$

Since $\left|\overline{S_{M}}\right|=m-i$, it follows that

$$
\left|\mathcal{B}_{2}\right|=\left(\left(\begin{array}{c}
m-i \\
k-(m-i)
\end{array}\right)\right)=\left(\begin{array}{c}
k-1 \\
k-m+i
\end{array}\right) .
$$

To show that $\left|\mathcal{A}^{\prime}\right|>|\mathcal{A}|$, it is sufficient to show that

$$
\left(\begin{array}{c}
k-1 \\
k-m+i
\end{array}\right)>\left(\begin{array}{c}
k-1 \\
k-i
\end{array}\right)
$$

or equivalently, that

$$
(k-i) !(i-1) !>(k-m+i) !(m-i-1) ! .
$$


Since $i<\frac{m}{2}$ and $m \leq k$, we have that $k-i>k-\frac{m}{2}>k-m+i \geq 1$. Therefore,

$$
\begin{aligned}
(k-i) !(i-1) ! & =(k-i)(k-i-1) \ldots(k-m+i+1)(k-m+i) !(i-1) ! \\
& \geq(m-i)(m-i-1) \ldots(i+1)(k-m+i) !(i-1) ! \\
& =\frac{m-i}{i}(m-i-1) !(k-m+i) ! \\
& >(m-i-1) !(k-m+i) !
\end{aligned}
$$

as required. Thus if $\mathcal{A}$ is of maximum size, it cannot contain a multiset with less than $\frac{m}{2}$ distinct elements from $[m]$.

It is easy to see that any $k$-multiset containing more than $\frac{m}{2}$ distinct elements from $[m]$ will intersect with any other such $k$-multiset. This completes the proof of the theorem for the case when $m$ is odd. When $m$ is even, it is necessary to consider the $k$-multisets which contain exactly $\frac{m}{2}$ distinct elements, that is, the $k$-multisets in $\mathcal{M}_{\left(\frac{m}{2}\right)}$. These multisets will intersect with any multiset containing more than $\frac{m}{2}$ distinct elements. However, $\mathcal{M}_{\left(\frac{m}{2}\right)}$ is not an intersecting collection. For any $A \in \mathcal{M}_{\left(\frac{m}{2}\right)}$, all of the $k$-multisets, $B$, where $S_{B}=\overline{S_{A}}$ will be in $\mathcal{M}_{\left(\frac{m}{2}\right)}$ and will not intersect with $A$. Since the size of a maximal intersecting collection of $\frac{m}{2}$-subsets of $[m]$ is $\frac{1}{2}\left(\begin{array}{c}m \\ \frac{m}{2}\end{array}\right)$ and each $\frac{m}{2}$-subset is the support for the same number of multisets in $\mathcal{M}_{\left(\frac{m}{2}\right)}$, an intersecting collection of $k$-multisets will contain at most half of the $k$-multisets in $\mathcal{M}_{\left(\frac{m}{2}\right)}$.

\section{Further work}

An obvious open problem is determining the size and structure of the largest collection of $t$-intersecting $k$-multisets, i.e. collections of multisets where the size of the intersection for every pair of multisets is at least $t$. (We define the intersection of two multisets to be the multiset containing all elements common to both multisets with repetitions.) The following conjecture is a version of Conjecture 5.1 from [4].

Conjecture 4.1. Let $k, m$ and $t$ be positive integers with $t \leq k$ and $m \geq t(k-t)+2$. If $\mathcal{A}$ is a collection of intersecting $k$-multisets of $[m]$, then

$$
|\mathcal{A}| \leq\left(\begin{array}{c}
m+k-t-1 \\
k-t
\end{array}\right) .
$$

Moreover, if $m>t(k-t)+2$, equality holds if and only if $\mathcal{A}$ is a collection of all the $k$-multisets from $[\mathrm{m}]$ that contain a fixed t-multiset from $[\mathrm{m}]$.

The lower bound on $m$ in this conjecture was obtained by substituting $m+k-1$ for $n$ in the corresponding bound for sets given by Frankl [8] and Wilson [14]. The conjecture is supported by the fact that when $m>t(k-t)+2$, a collection consisting of all $k$-multisets containing a fixed $t$-multiset is larger than a collection consisting of all $k$-multisets containing $t+1$ elements from a set of $t+2$ distinct elements of $[m]$ and that these two collections are equal in size when $m=t(k-t)+2$. Furthermore, when 
$m=t(k-t)+1$, collections larger than $\left(\begin{array}{c}m+k-t-1 \\ k-t\end{array}\right)$ are possible. For example, if $t=2$, $k=5$ and $m=7$, the cardinality of the collection of all $k$-multisets containing three or more elements from $\{1,2,3,4\}$ is 91 while $\left(\begin{array}{c}m+k-t-1 \\ k-t\end{array}\right)=84$.

The existence of a graph homomorphism from the Kneser graph $K(n, k)$ to its multiset analogue $M(m, k)$ in the proof of Theorem 1.2 gave a simple and straight-forward way to show that the size of the largest independent set in $M(m, k)$ is no larger than the size of the largest independent set in $K(n, k)$. These graphs can be generalized as follows: let $K(n, k, t)$ be the graph whose vertices are the $k$-subsets of $[n]$ and where two vertices, $A, B$, are adjacent if $|A \cap B|<t$ and let $M(m, k, t)$ be the graph whose vertices are the $k$-multisets of $[m]$ and where two vertices, $C, D$ are adjacent if $|C \cap D|<t$.

If a bijective homomorphism from $K(n, k, t)$ to $M(m, k, t)$ exists, it could be used to prove a bound not only on the maximum size of a $t$-intersecting collection as given in Conjecture 4.1 but also on the maximum size when $k-t \leq m \leq t(k-t)+2$ using the Complete Erdős-Ko-Rado theorem of Ahlswede and Khachatrian [1]. However, it is not clear that such a homomorphism exists. The conditions placed on the bijection in the proof of Theorem 1.2 are not sufficient to ensure that the bijection is a homomorphism since for two $k$-multisets, $A$ and $B$, having $\left|S_{A} \cap S_{B}\right|<t$ does not imply that $|A \cap B|<t$.

The simple fact that if a graph $G$ is isomorphic to a spanning subgraph of a graph $H$, then $\alpha(H) \leq \alpha(G)$ may be useful in proving Erdős-Ko-Rado theorems for different objects. It would be interesting to determine if there are combinatorial objects other than multisets which have this relationship to an object for which an Erdős-Ko-Rado type result is known.

\section{Acknowledgment}

We are grateful for the helpful comments of the anonymous referee, particularly those concerning Theorem 1.3 which greatly simplified the proof.

\section{References}

[1] R. Ahlswede and L.H. Khachatrian. The complete intersection theorem for systems of finite sets. European J. Combin., 18(2):125-136, 1997.

[2] I. Anderson. An Erdős-Ko-Rado theorem for multisets. Discrete Math., 69(1):1-9, 1988.

[3] I. Anderson. Combinatorics of finite sets. Dover Publications Inc., Mineola, NY, 2002.

[4] G. Brockman and B. Kay. Elementary techniques for Erdős-Ko-Rado-like theorems. arXiv: 0808.0774, August 2008.

[5] P. Erdős, M. Herzog, and J. Schönheim. An extremal problem on the set of noncoprime divisors of a number. Israel J. Math., 8:408-412, 1970.

[6] P. Erdös, Chao Ko, and R. Rado. Intersection theorems for systems of finite sets. Quart. J. Math. Oxford Ser. (2), 12:313-320, 1961. 
[7] P. Erdős and J. Schönheim. On the set of non pairwise coprime divisors of a number. In Combinatorial theory and its applications, I (Proc. Colloq., Balatonfüred, 1969), pages 369-376. North-Holland, Amsterdam, 1970.

[8] P. Frankl. The Erdős-Ko-Rado theorem is true for $n=c k t$. In Combinatorics (Proc. Fifth Hungarian Colloq., Keszthely, 1976), Vol. I, volume 18 of Colloq. Math. Soc. János Bolyai, pages 365-375. North-Holland, Amsterdam, 1978.

[9] P. Frankl and Z. Füredi. The Erdős-Ko-Rado theorem for integer sequences. SIAM J. Algebraic Discrete Methods, 1(4):376-381, 1980.

[10] P. Frankl and N. Tokushige. The Erdős-Ko-Rado theorem for integer sequences. Combinatorica, 19(1):55-63, 1999.

[11] H.-D. O. F. Gronau. More on the Erdős-Ko-Rado theorem for integer sequences. $J$. Combin. Theory Ser. A, 35(3):279-288, 1983.

[12] G. O. H. Katona. A simple proof of the Erdős-Chao Ko-Rado theorem. J. Combinatorial Theory Ser. B, 13:183-184, 1972.

[13] M. Mahdian. private communication, 2010.

[14] R.M. Wilson. The exact bound in the Erdős-Ko-Rado theorem. Combinatorica, $4(2-3): 247-257,1984$. 